\title{
Are the compact star clusters in M82 evolving towards globular clusters?
}

\author{
Y.D. Mayya, D. Rosa-González, L. Rodríguez, L. Carrasco, R. Romano and \\ A. Luna \\ Instituto Nacional de Astrofísica, Optica y Electronica, Luis Enrique Erro 1, \\ Tonantzintla, C.P. 72840, Puebla, Mexico ydm@inaoep.mx \\ Summary. ${ }^{1}$ Recent HST/ACS images of M82 covering the entire galaxy have been \\ used to detect star clusters. The galaxy is known to contain a young population \\ (age $<10^{7} \mathrm{yr}$ ) in its starburst nucleus, surrounded by a post-starburst disk of age \\ $<10^{9}$ yr. We detect more than 650 star clusters in this galaxy, nearly 400 of them \\ in the post-starburst disk. These data have been used to derive the luminosity, \\ mass and size functions separately for the young nuclear, and intermediate-age disk \\ clusters. In this contribution, we discuss the evolutionary status of these clusters, \\ especially, on the chances of some of these clusters surviving to become old globular \\ clusters.
}

\section{Introduction}

Super star clusters (SSCs) and globular clusters (GCs) represent the youngest and the oldest stellar aggregates known in the Universe. The environments in which these two kinds of clusters are found are vastly different - SSCs are found in violent star-forming regions, whereas GCs are found in the halos of galaxies. Yet, the similarity in their compactness and mass, is a reason compelling enough to think of an evolutionary connection between them. The growing popularity of the hierarchical model of galaxy formation in the years following the discovery of SSCs, and the possibility of observing the epochs of galaxy (and GC) formation at high redshifts, have also generated interest in looking for a common origin for these two seemingly different classes of clusters.

In order to investigate the relation between the two types of clusters, it is important to analyze the survival of SSCs for a Hubble time. Star clusters are vulnerable to a variety of disruption processes that operate on three

\footnotetext{
${ }^{1}$ To appear in proceedings of the Puerto Vallarta Conference on "New Quests in Stellar Astrophysics II: Ultraviolet Properties of Evolved Stellar Populations" eds. M. Chavez, E. Bertone, D. Rosa-Gonzalez \& L. H. Rodriguez-Merino, Springer, ASSP series.
} 
different timescales (see Fall \& Zhang, 2001; Maíz-Apellániz, 2004; de Grijs $\&$ Parmentier, 2007, for more details). On short timescales $\left(t \sim 10^{7} \mathrm{yr}\right)$, the exploding supernovae and the resulting superwinds are responsible for cluster expansion and disruption, a process popularly dubbed as infant mortality. On intermediate timescales $\left(10^{7}<t<\right.$ few $\left.\times 10^{8} \mathrm{yr}\right)$, the mass-loss from evolving stars leads to the disruption of the clusters. On even longer timescales $\left(t>\right.$ few $\times 10^{8}$ yr $)$, stellar dynamical processes, especially evaporation due to two-body scattering, and tidal effects on a cluster as it orbits around the galaxy, known as gravitational shocks, come into play in the removal of stellar mass from clusters. The GCs represent those objects that have survived all these processes, whereas young SSCs are just experiencing them. Intermediate age SSCs are the ideal objects to investigate the influence of disruption processes on the survival of star clusters. Almost all the star formation in the disk of M82 took place in a violent disk-wide burst around 100-500 Myr ago, following the interaction of M82 with the members of M81 group (Mayya et al., 2006). Cluster formation is known to be efficient during the burst phase of star formation (Bastian et al., 2005), and hence we expect large number of clusters of intermediate age $(\sim 100 \mathrm{Myr})$ in its disk. Hence, M82 offers an excellent opportunity to assess the evolutionary effects on the survival of star clusters, and to look for a possible evolutionary connection between the SSCs and GCs.

\section{Data Extraction, Source Selection, and Simulations}

The observational data used in this work consisted of images in $\mathrm{F} 435 \mathrm{~W}(B)$, F555W $(V)$ and F814W (I) filters, that were obtained by the Hubble Heritage Team (Mutchler et al., 2007) using the ACS/WFC instrument aborad the Hubble Space Telescope (HST). Bias, dark, and flat-field corrections were carried out using the standard pipeline process by the Heritage Team. The final reduced science quality images cover the entire optical disk of the galaxy with a spatial sampling of 0.05 arcsec pixel $^{-1}$, which corresponds to 0.88 parsec pixel ${ }^{-1}$ at M82's distance of 3.63 Mpc (Freedman et al., 1994). The point sources have a size distribution that peaks at a Full Width at Half Maximum (FWHM) of 2.1 pixels, with the tail of the distribution extending to 3.0 pixels (or 2.6 parsec). Very few clusters are expected to have sizes smaller than 3 parsec, and hence clusters can be distinguished from the stars on these images. A circle of 500 pixels $(450 \mathrm{pc})$ radius is used to separate the nuclear region from the disk. The clusters inside this radius are associated with strong $\mathrm{H} \alpha$ emitting complexes, and hence are younger than 10 Myr (Melo et al., 2005). On the other hand, the disk outside the $450 \mathrm{pc}$ radius shows characteristic signatures of post-starburst conditions, with hardly any $\mathrm{H} \alpha$ emission.

We used SExtractor (Bertin \& Arnouts, 1996) independently on the $B, V$, and $I$-band images to construct an unbiased sample of cluster candidates. A source having a FWHM $>3$ pixels and an area of at least 50 adjacent pixels, 
each of $\mathrm{S} / \mathrm{N}>5$ is considered a cluster candidate. All the bright sources satisfying these criteria are genuine clusters, but at fainter magnitudes majority of the candidate sources lack the symmetry expected for a physical cluster. These are found to be artificial extended sources formed due to the superposition of stars in this nearly edge-on galaxy. These artificial sources most often are elongated, and are rejected automatically from the sample using the ellipticity parameter of SExtractor. Cluster candidates in each filter were then combined, the common sources being counted only once. The resulting list contains 653 clusters, 260 of them belonging to the nuclear region. For all the sources in the final list, aperture photometry is carried out in all the three bands. The FWHM calculated by SExtractor is used as a measure of the size of the clusters.

The observed cluster luminosity function (LF) follows a power-law at the bright end, turning over sharply at faint magnitudes. Similarly, the size distribution function peaks at a characteristic value of FWHM 10 pixels. Monte Carlo simulations were carried out to check the effect of incompleteness of cluster detection on the observed functional forms. In the simulations, each cluster is assumed to be round and to have a Gaussian intensity profile of a given FWHM. A power-law distribution function is used to assign a luminosity to each cluster. Two separate simulations are done, one in which a cluster is assigned a size based on a power-law size distribution function, and the other based on a log-normal function. The simulated luminosity function resembles very much the observed one, implying that the observed turn-over of the luminosity function is due to incompleteness at the faint end and not intrinsic to the cluster population. Hence, the turn-over in the luminosity function, if any, would correspond to a magnitude fainter than $B=22$ mag. On the other hand, the observed size function points to an intrinsically log-normal size distribution, rather than a power-law function. A more detailed description of the selection process, observed luminosity and size functions, and the Monte Carlo simulations can be found in Mayya et al. (2007).

\section{Physical Parameters of Clusters}

We analyzed the color and magnitude of the individual clusters to obtain their reddening and mass, making use of solar metallicity Single Stellar Population (SSP) models of Girardi et al. (2002). These authors provide the evolutionary data on colors and magnitudes for the instrumental HST/ACS filters, a fact that enables us a direct comparison with the observed data. The Kroupa (2001) initial mass function (IMF) in its corrected version has been used. It has nearly a Salpeter slope (2.30 instead of 2.35) for all masses higher than $1 M \odot$. The derived masses depend on the assumption of the lower cut-off mass of the IMF. In the case of standard Kroupa's IMF, the derived masses would be around 2.5 times higher. 


\subsection{The Ages of Clusters}

Colors obtained by the combination of the three filters that we used suffer

from age-reddening degeneracy, and hence it was necessary to assume one of the quantities to obtain the other. We found that the observed range in colors is too large to be explained by evolutionary effects, even for stellar populations as old as $10 \mathrm{Gyr}$. On the other hand, age of the principal stellar populations in the nuclear region of M82 is determined in innumerable studies (Rieke et al., 1993; Förster Schreiber et al., 2003, and references therein), and it is found to be $<10 \mathrm{Myr}$. Based on these studies, we adopt an age of $8 \mathrm{Myr}$ for the nuclear clusters. Most of the disk stars in M82 were formed in a violent burst around $500 \mathrm{Myr}$ ago. Ages of those clusters for which spectroscopic data are available (Smith et al., 2006) lie in the range between 50-500 Myr, suggesting that the clusters are formed during or immediately after the disk-wide star formation epoch.

\subsection{Color-Magnitude Diagrams}

From the assumed ages ( 8 Myr for the nuclear clusters, and 50-500 Myr for the disk clusters), and the very likely hypothesis that the extinction is the main cause of the dispersion in the observed colors, we can estimate the masses of the clusters. The method we have followed is illustrated in Figure 1. For a given position in the Color Magnitude Diagram (CMD), we derived the extinction by comparing the observed colors with those of the SSP. Once the extinction is determined, we calculate the mass using the extinction-corrected luminosity and the mass-to-light ratio of such SSP. The disk masses are derived assuming an age of 100 Myr. The mass estimates would be higher by a factor of 3.2, if the clusters are as old as the stellar disk (500 Myr). On the other hand, if the clusters are as young as $50 \mathrm{Myr}$, the masses would be lower by a factor of 1.6. The distribution of the derived visual extinction values is peaked at $\sim 1 \mathrm{mag}$ for the disk clusters, whereas it is flat between 1-4 mag for the nuclear clusters.

\subsection{Mass Distribution Function}

The determination of the cluster masses for our complete sample enables us to derive the present-day Cluster Mass Function (CMF). In the left panel of Figure 2, we plot the CMF separately for the nuclear and disk clusters. The nuclear CMF is scaled up to match the disk CMF at $1.5 \times 10^{6} M \odot$. Poissonian error bars are indicated. The distribution for both samples follows a powerlaw over almost two orders of magnitude in mass for cluster masses above $\sim 2 \times 10^{4} M \odot$. However, the power-law index for the disk and nuclear cluster populations shows a clear difference, $\alpha=1.8 \pm 0.1$ for the nuclear clusters, and $1.5 \pm 0.1$ for the disk population. Studies of young star clusters in nearby 

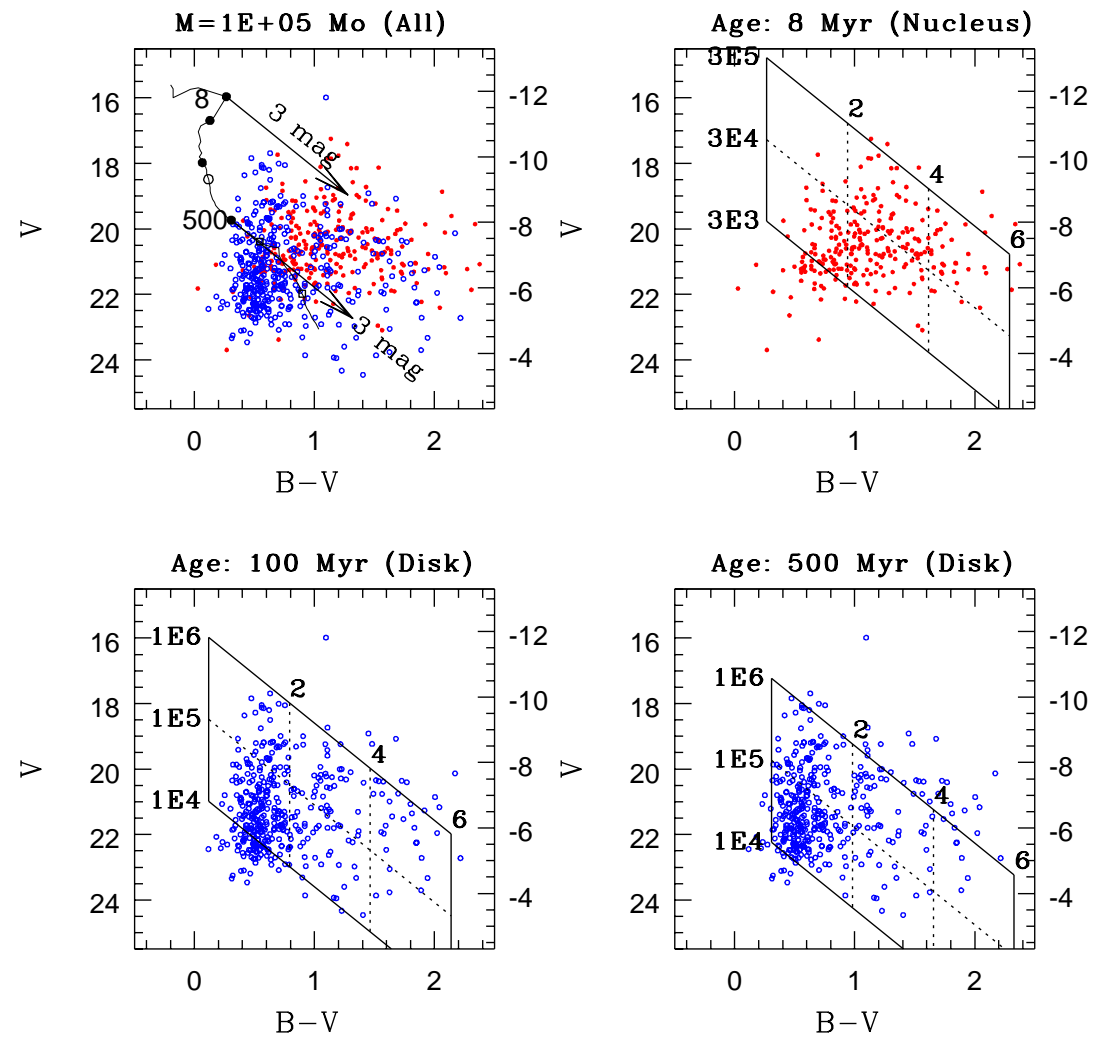

Fig. 1. Observed color-magnitude diagrams (CMDs) for the nuclear (filled circles) and disk clusters, in M82. (Top left) Evolutionary track for an SSP of a cluster mass of $10^{5} M \odot$ is superposed. Two vectors, placed at $8 \mathrm{Myr}$ and $500 \mathrm{Myr}$, show the location of the track reddened by $A_{v}=3 \mathrm{mag}$. In the top-right panel, we show the CMD for the nuclear clusters only. The locations of an 8 Myr SSP for a range of cluster masses and visual extinctions are shown by the superposed grid. Mass varies vertically along the grid (in solar units), whereas the visual extinction (in magnitude) varies along the diagonal axis. In the bottom panels, we show a similar diagram for the disk clusters, with the superposed grids corresponding to fixed ages of 100 Myr (left) and 500 Myr (right). In all the panels, tick mark values of the right-vertical axis correspond to the absolute magnitude in the $V$-band. 

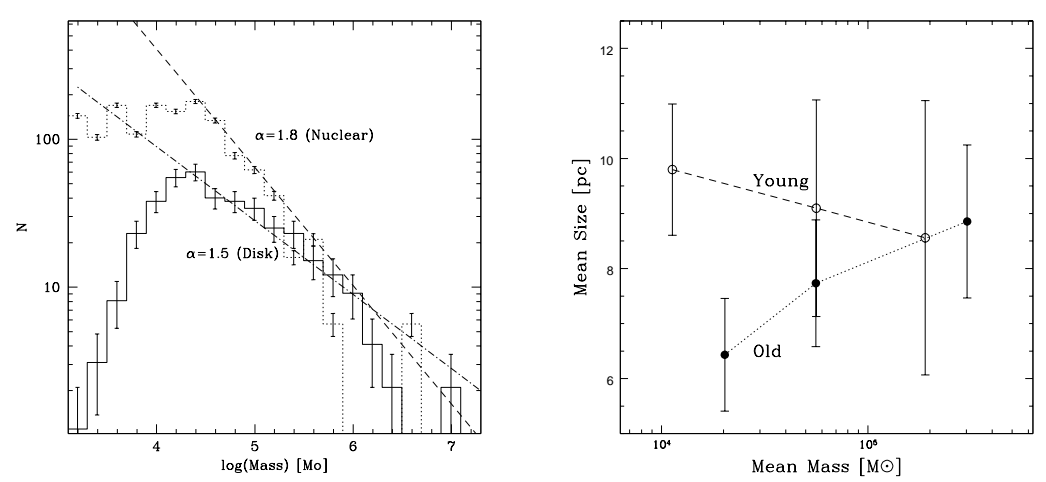

Fig. 2. (Left) Mass functions for the nuclear (dotted line) and disk (solid line) cluster samples. Both the samples follow a power-law distribution between $2 \times 10^{4} \mathrm{M} \odot$ and $10^{6} M \odot$. The best-fit indices in this mass range are indicated. (Right) Mean size (FWHM) of the clusters as a function of mean mass for three mass bins for the nuclear (young) and disk (old) samples. The error bar denotes the rms dispersion about the mean value. High mass clusters have similar mean sizes irrespective of their evolutionary status. On the other hand, mean size of the low-mass clusters clearly decreases as they become older. Among the young clusters, low-mass ones are more extended than higher mass ones.

galaxies yield a value of $\alpha$ close to 2.0 (de Grijs et al., 2003). Hence, $\alpha=2.0$ can be considered as the expected slope of the initial CMF.

In general, the cluster size distribution function (CSF) for the nuclear and disk clusters follow a log-normal form. However, the mean, as well as the maximum cluster sizes are systematically smaller for the lower mass bins. This tendency is illustrated in the right panel of Figure 2, where the mean cluster size for each mass bin has been plotted against the mean mass of clusters in that bin, for the young and old ones, separately. For the highest mass bin, the mean sizes of the young and old clusters are similar. The mean size decreases systematically with decreasing cluster mass for the old clusters, whereas the inverse is true for the young clusters.

\section{On the Survival Chances of Star Clusters in M82}

The observed differences in the CSF for young and old clusters are consistent with the expected evolutionary effects. Both the disruption of the loose OB associations and the dynamical trend towards relaxation would diminish the number of large low-mass systems. Thus, the destruction process is both mass and size dependent, with the most extended clusters in each mass bin being the most vulnerable to disruption. All clusters of masses higher than $10^{5} \mathrm{M} \odot$ are 
still surviving $\sim 10^{8}$ yr after their formation. In this section, we discuss these observational results in the context of theoretical models of cluster disruption, and their possible survival to become globular clusters.

At early times, disruption is caused mainly due to the expulsion of the intra-cluster gas through supernova explosions. This process is ineffective once all the high mass stars in the cluster die, which happens in around $\sim 30 \mathrm{Myr}$. Hence, the observed disk clusters have survived this early mechanism of disruption. On intermediate timescales $\left(10^{7}<t<\right.$ few $\left.\times 10^{8} \mathrm{yr}\right)$, the mass-loss from evolving stars leads to a decrease in the cluster mass from its initial value. Clusters can loose as much as $30 \%$ of their stellar mass during their evolution. The decreased cluster mass can result in the expansion of the cluster, finally leading to its disruption. However, this process of disruption is equally effective for high and low mass clusters, and hence a change in the slope of mass function is not expected. The observed flattening of the mass function at older ages, suggests that the cluster disruption process that is active in M82 selectively destroys low-mass clusters. The tidal effect experienced by the clusters as they move in the gravitational field of the parent galaxy is one such process. According to Fall \& Zhang (2001), this process becomes important after $\sim 300 \mathrm{Myr}$ in normal galaxies. However, in the case of M82, de Grijs et al. (2005) have estimated a disruption timescale as short as $30 \mathrm{Myr}$ for a cluster of mass $10^{4} M \odot$ at $1 \mathrm{kpc}$ away from the center, with a dependence on mass that varies as $M^{0.6}$. The short timescale in M82 implies that the surviving clusters in the disk are presently experiencing the dynamical processes of cluster disruption.

If the trend of selective disruption of loose clusters continues, how many of the present clusters will survive for a Hubble time? Can the LF of the surviving clusters look like that of the Galactic GCs? In Figure 3, we show the evolutionary effects on the LF of the M82 clusters. The histogram with dashed lines shows the LF considering the photometric evolution of the clusters for 5 Gyr, whereas the solid histogram shows the same, but after taking into account the dynamical effects as well. The latter is implemented in a simplistic way, by imposing the condition that for the clusters to survive the dynamical effects, their half-light radius, $R_{\text {eff }}$, should be smaller than the tidal radius, $R_{\mathrm{t}}$, for that cluster. For a cluster of mass $M_{\mathrm{C}}$ at galactocentric radius $R_{\mathrm{G}}, R_{\mathrm{t}}$ is given by the expression (Spitzer, 1987),

$R_{\mathrm{t}}=\left(\frac{M_{\mathrm{C}}}{2 M_{\mathrm{G}}}\right)^{1 / 3} R_{\mathrm{G}}$,

where $M_{\mathrm{G}}$ is the mass of the parent galaxy, which for M82 is somewhat uncertain due to the difficulty in interpreting uniquely the observed gas velocity fields in this disturbed galaxy, and the best estimate is $M_{\mathrm{G}}=10^{10} M \odot$ (Sofue , 1998). The $R_{\mathrm{t}}$ values calculated using the currently observed galactocentric distances indicate that most of the nuclear clusters, and very few of the disk clusters, will be destroyed. Thus, if the clusters are in circular orbits, the LF will practically retain its present power-law form. However, the stellar orbits in the central bar of M82 are known to be highly elliptical (Greve et al. , 2002), 
which implies that the galactocentric distance of a cluster will change with time. The disruption of a cluster depends on the net tidal force received by its stars as it orbits the galaxy during its lifetime. We found that for an assumed $R_{\mathrm{G}}=350 \mathrm{pc}$, the future LF of M82 will resemble that of the Galactic GCs. Even in this extreme case, 85 clusters will survive, as compared to the 146 GCs in the Milky Way. The number of GCs in a galaxy scale with the mass of the parent galaxy, and considering that M82 is an order of magnitude less massive than the Milky Way, only 15 GCs are expected to present in M82. Thus, number of clusters that will survive represent an over-abundance by a factor of 5-6. For ages older than this, the distribution is similar except that the peak of the distribution shifts to $\sim 0.5$ mag fainter. Thus, the compact star clusters in M82 will evolve into GCs.

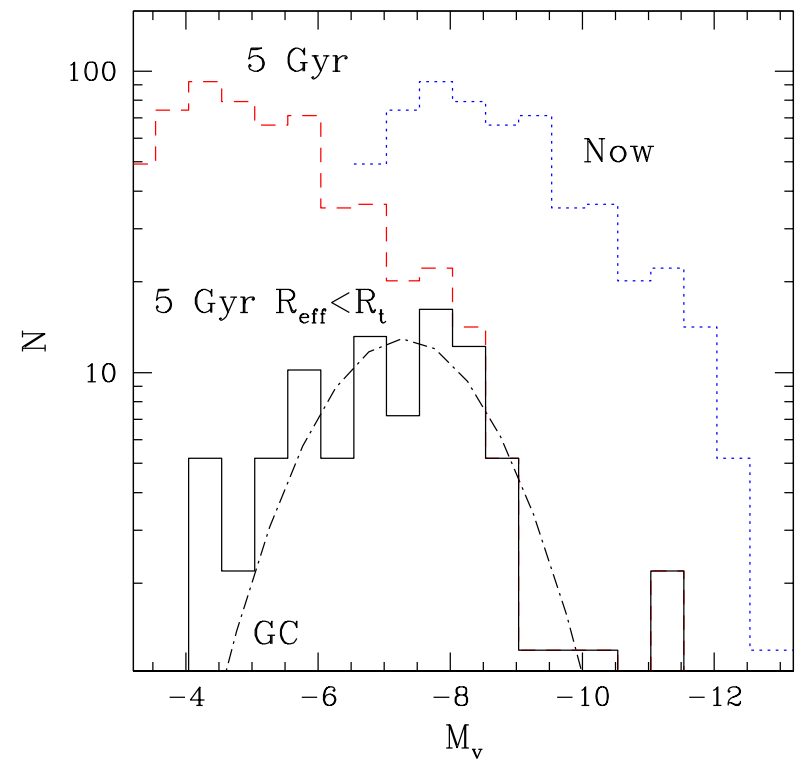

Fig. 3. Present (dotted histogram) and future luminosity functions of M82 star clusters with (solid histogram) and without (dashed histogram) taking into account dynamical effects of evolution at an age of 5 Gyr. The log-normal function representing the luminosity function of the Galactic Globular Clusters is shown by the dot-dashed curve. 


\section{Conclusions}

Luminosity and Mass functions of star clusters in M82 follow power-law functions, with the power law index showing a tendency for flattening of the profile with age. In other words, there is a deficiency of low-mass clusters among the older clusters. We also find the mean size of the older clusters to be smaller as compared to the younger clusters for masses $<10^{5} M \odot$. These two results together imply the selective destruction of loose clusters. The tidal forces experienced by the clusters as they orbit around the galaxy lead to exactly such a destruction process. If this process continues in M82, the LF of surviving clusters can mimic the presently observed LF of the Galactic GCs, provided the clusters move around the galaxy in highly elliptical orbits, with perigalactic distance as small as $350 \mathrm{pc}$. The resulting LF contains 85 clusters with the function peaking at the same luminosity as for the Galactic GCs at 5 Gyr age, and fainter by $\sim 0.5 \mathrm{mag}$ at $10 \mathrm{Gyr}$. On the other hand, if the clusters move in nearly circular orbits, the LF will retain the power-law form, with the number of surviving clusters even higher.

This work is partly supported by CONACyT (Mexico) research grants 42609-F and 49942-F. We would like to thank the Hubble Heritage Team at the Space Telescope Science Institute for making the reduced fits files available to us.

\section{References}

Bastian, N., Gieles, M., Lamers, H. J. G. L. M., Scheepmaker, R. A., \& de Grijs, R. 2005, A\&A, 431, 905

Bertin, E., \& Arnouts, S. 1996, A\&AS, 117, 393

de Grijs, R., Anders, P., Bastian, N., Lynds, R., Lamers, H. J. G. L. M., \& O'Neil, E. J. 2003, MNRAS, 343, 1285

de Grijs, R., Parmentier, G., \& Lamers, H. J. G. L. M. 2005, MNRAS, 364, 1054

de Grijs, R., \& Parmentier, G. 2007, Chinese Journal of Astronomy and Astrophysics, 7, 155

Fall, S. M., \& Zhang, Q. 2001, ApJ, 561, 751

Förster Schreiber, N. M., Genzel, R., Lutz, D., \& Sternberg, A. 2003, ApJ, 599, 193

Freedman, W. L., et al. 1994, ApJ, 427, 628

Girardi, L., Bertelli, G., Bressan, A., Chiosi, C., Groenewegen, M. A. T., Marigo, P., Salasnich, B., \& Weiss, A. 2002, A\&A, 391, 195

Greve, A., Wills, K. A., Neininger, N. \& Pedlar, A. A\&A, 383, 56

Kroupa P., 2001, MNRAS 322, 231

Maíz-Apellániz, J. 2004, Astrophysics and Space Science Library, 315, 231 
Mayya, Y. D., Bressan, A., Carrasco, L., \& Hernandez, L. 2006, ApJ, 649, 172

Mayya, Y. D., Romano, R., Rodríguez-Merino, L.H., Luna, A., Carrasco, L. \& Rosa-González, D. 2007, ApJ submitted

Melo, V. P., Muñoz-Tuñón, C., Maíz-Apellániz, J., \& Tenorio-Tagle, G. 2005, ApJ, 619, 270

Mutchler, M., Bond, H. E., Christian, C. A., Frattare, L. M., Hamilton, F., Januszewski, W., Levay, Z. G., Mountain, M., Noll, K. S., Royle, P., Gallagher, J. S., \& Puxley, P. 2007, PASP, 119, 1

O'Connell, R. W., Gallagher, J. S., III, Hunter, D. A., \& Colley, W. N. 1995, ApJL, 446, L1

Rieke, G. H., Loken, K., Rieke, M. J., \& Tamblyn, P. 1993, ApJ, 412, 99

Smith, L. J., Westmoquette, M. S., Gallagher, J. S., O'Connell, R. W., Rosario, D. J., \& de Grijs, R. 2006, MNRAS, 370, 513

Sofue, Y. 1998, PASJ, 50, 227

Spitzer, L. 1987, Princeton, NJ, Princeton University Press, 1987, p15 